\title{
MEDICAL PHYSICISTS' IMPLICATION IN RADIOLOGICAL DIAGNOSTIC PROCEDURES: RESULTS AFTER 1 Y OF EXPERIENCE
}

Nick Ryckx*, Silvano Gnesin, Reto Meuli, Christel Elandoy and Francis R. Verdun

Institute of Radiation Physics, Lausanne University Hospital, Rue du Grand-Pré 1, Lausanne CH-1007, Switzerland

*Corresponding author: nick.ryckx@chuv.ch

\begin{abstract}
Since January 2008 - de facto 2012 - medical physics experts (MPEs) are, by law, to be involved in the optimisation process of radiological diagnostic procedures in Switzerland. Computed tomography, fluoroscopy and nuclear medicine imaging units have been assessed for patient exposure and image quality. Large spreads in clinical practice have been observed. For example, the number of scans per abdominal CT examination went from 1 to 9. Fluoroscopy units showed, for the same device settings, dose rate variations up to a factor of 3 to 7 . Quantitative image quality for positron emission tomography (PET)/CT examinations varied significantly depending on the local image reconstruction algorithms. Future work will be focused on promoting team cooperation between MPEs, radiologists and radiographers and on implementing task-oriented objective image quality indicators.
\end{abstract}

In 1997, the European Council Directive 97/43/ EURATOM introduced the implication of medical physics experts (MPEs) in the optimisation process of radiological diagnostic procedures. That recommendation has been translated into the Swiss Radiological Protection Ordinance $\left(\mathrm{RO}^{(1)}\right)$ and applied officially in 2008, de facto in 2012. This contribution summarises the application methodology, as well as the results, after 1 y of experience. (NB: The Radiological Protection Ordinance is usually abbreviated using RPO, but RO will be used in this text to avoid any confusion with 'Radiation Protection Officer'.)

\section{INTRODUCTION}

In Switzerland, the Federal Office of Public Health (FOPH) legally authorises the manufacturer for performing quality control tests of their radiological devices. The minimal frequency, as well as the contents of those periodical controls, is set by the FOPH.

The manufacturer ensures that the device runs in accordance with the norms and criteria established by the International Electrotechnical Commission (IEC) standards. As a consequence, until now, the tasks of the manufacturer were completely separated from those of the users (physicians and radiographers).

In addition to these tests, the FOPH audits the different radiological centres on a regular basis, in order to ensure compliance with the national and international safety and quality standards.

Until now, no MPE was involved in the optimisation process in diagnostic and therapeutic radiological procedures. As such, the manufacturer is judge and jury of the implemented clinical protocols. The authors' aim is to provide objective image quality assessment tools in order to use the devices to their full potential in dose optimisation while remaining clinically relevant. As such:

- The manufacturer will continue to maintain the technical integrity of the device, ensuring the link between detector dose and image quality.

- The radiation protection expert is in charge of the operational safety of the staff using the device.

- The MPE, in close collaboration with the physicians and radiographers, will make sure that the device is being properly used on patients, ensuring the link between patient dose and image quality (or, more specifically, diagnostic quality).

In opposition to several countries where MPEs are already in place, a lot had to be done to rethink the role of the MPE. The MPEs' work had to yield added value to the clinical practice without substituting the manufacturer for the periodical technical controls.

The major part of the authors' effort was put in the field of computed tomography (CT), since it is the most common diagnostic device in the vast majority of private and public radiology practices. Furthermore, the radiation protection requirements in interventional radiology and nuclear medicine (NM) being more obvious, the involvement of an MPE was much easier in these fields.

\section{OBJECTIVES AND METHODOLOGY}

The authors will now describe the approach used for the implementation of Article 74, Paragraph 7, of the RO. The description, as well as the rest of this contribution, will be separated into three imaging fields: CT, fluoroscopy and NM.

A common objective for those three fields was to make an inventory of the different devices and 


\section{N. RYCKX ET AL.}

technologies used by the centres in the authors' database, as well as gaining acceptance within the different radiology institutes.

\section{Computed tomography}

The approach used for CT was focused on three axes:

- verification of the main dose indicators, since they are used as the primary tools for protocol optimisation,

- benchmarking of the institutions' image quality using a pre-defined acquisition and reconstruction protocol on a QA phantom,

- dosimetry assessment of the clinical use of the devices.

\section{Machine output}

First: control of the main output parameters of the device itself:

- volumetric computed tomography dose index $\left(\mathrm{CTDI}_{\mathrm{vol}}\right)$, using a standard IEC 16-cm-diameter head phantom and a $100-\mathrm{mm}$ pencil ion chamber (Radcal Corporation, Monrovia, California),

- beam collimation and penumbra, using selfdeveloping Gafchromic XR-CT strips,

- Hounsfield unit (HU) calibration in water at the available X-ray tube voltages,

- laser alignment using a Catphan ${ }^{\circledR} 600$ aligned on the lasers, later on compared with the projection image (topogram).

\section{Local abdomen protocol qualification and unit benchmarking for a given CTDI $I_{\text {vol }}$ condition}

Second: qualification of the local abdomen acquisition and image reconstruction protocols using a standard QA phantom (Catphan ${ }^{\circledR} 600$, The Phantom Laboratory, Salem, New York). Furthermore, a standard acquisition and reconstruction protocol was elaborated in order to enable device benchmarking. Standard test parameters were as follows:

- $\mathrm{CTDI}_{\mathrm{vol}}$ of $15 \mathrm{mGy}$, corresponding to the Swiss diagnostic reference level (DRL) for an abdominal examination,

- gantry rotation time of $1 \mathrm{~s}$,

- reconstructed slice thickness of $2.5 \mathrm{~mm}$ (or $2 \mathrm{~mm}$, depending on the CT manufacturer and/or software version), with contiguous slices,

- standard (soft tissue) reconstruction kernel,

- filtered back-projection, no iterative reconstruction,

- field of view (FOV) of $220 \mathrm{~mm}$.

As body examinations yield the largest effective $\operatorname{doses}^{(2)}$ with the largest spread in clinical practice ${ }^{(3)}$, one of the critical aspects in diagnosis is the low-contrast detectability (LCD). This depends strongly on the used dose, the reconstruction convolution kernel, the slice thickness and the detector performance. The three first parameters being equal, the latter could be benchmarked. For this purpose, three observers have looked at the images on a diagnostic screen and estimated subjectively the diameter of the smallest rod they could distinguish for a given contrast.

\section{Clinical use of the device}

Third: dosimetry analysis of a sample of clinical examinations sent by the different institutions. The requested examinations concerned standard head, chest, abdomen, paediatric, upper- and lower-limb and cardiac acquisitions. The analysed parameters were as follows:

- $\mathrm{CTDI}_{\mathrm{vol}}$ per scan,

- cumulated dose-length product (DLP), as well as DLP per scan,

- examination effective dose $E^{(2)}$,

- number of scans per examination,

- measurement of the antero-posterior and lateral (LAT) patient dimensions, to calculate the sizespecific dose estimate $^{(4)}$,

- used slice thicknesses,

- noise in a homogeneous zone for a given slice thickness,

- assessment of the use of the automatic exposure control system,

- positioning of the patient in the gantry,

- visibility check of a given list of organs by review of the diagnostic images by an experienced radiographer.

\section{Fluoroscopy}

Fluoroscopy units were characterised using the locally implemented clinical parameters. The characterisation was done using PMMA slabs of different thicknesses, thus simulating patients of different sizes (Figure 1). Dose rates measured in different sites and units were collected and compared.

Since the dose rates of different units can differ significantly ${ }^{(5)}$, the aim was to provide the users of the unit with a framework that showed the orders of magnitude of their patients' exposure by their most commonly used clinical protocols.

No image quality measurements were performed. However, clinical image quality requirements were apparently satisfied for all systems since physicians use those units without any preference.

\section{Nuclear medicine}

Quantitative imaging is of prime importance in NM. Indeed, the estimation of the volumetric activity in a lesion will yield precious information about its metabolism and will influence the subsequent clinical decisions. This is why these aspects have to be assessed correctly. 


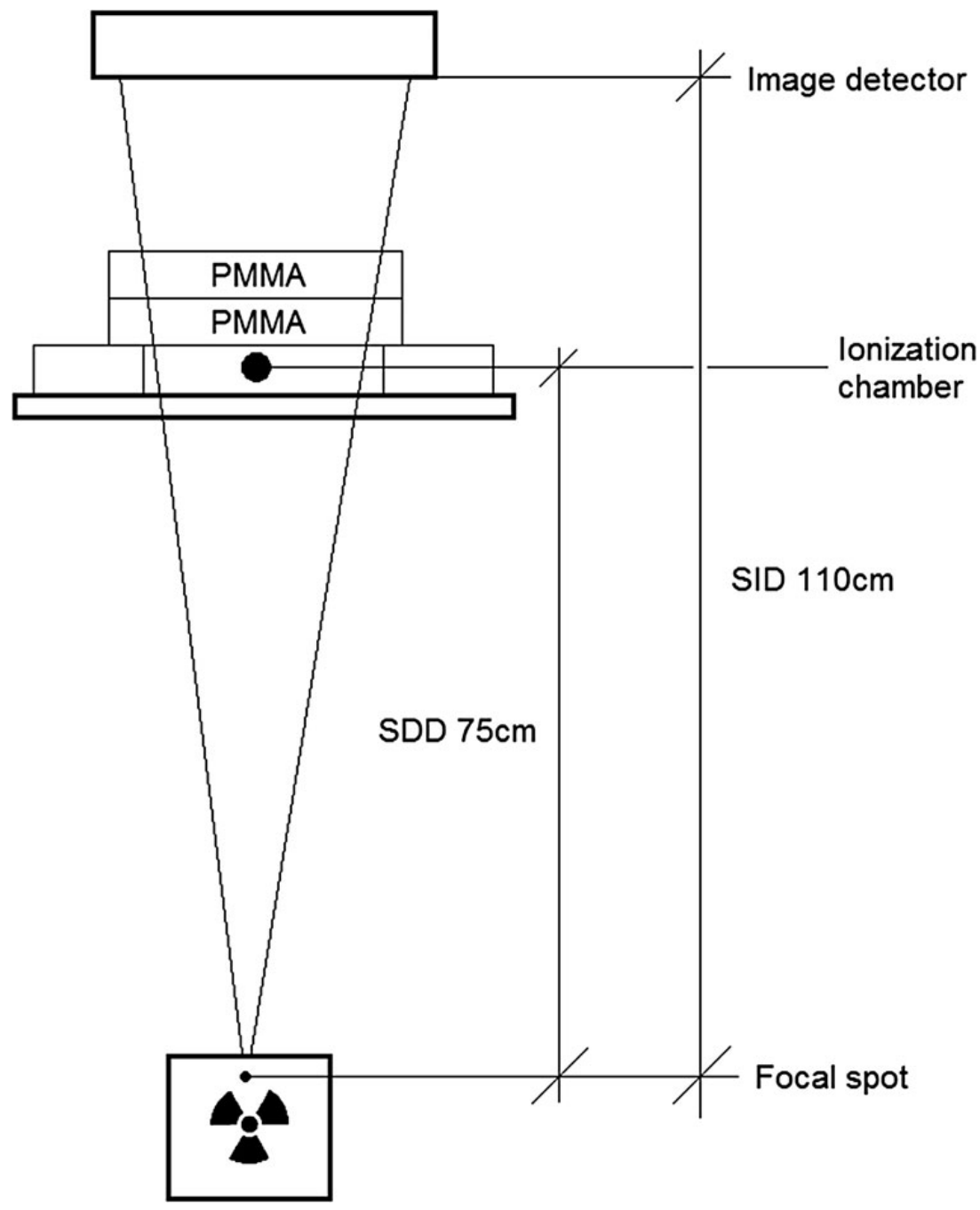

Figure 1. Schematic figure of the fluoroscopy characterisation geometry: PMMA in the primary beam, ion chamber at the entrance point of the beam. Source-to-imaging device distance (SID): $110 \mathrm{~cm}$, source-to-detector distance (SDD): $75 \mathrm{~cm}$.

\section{Single photon emission computed tomography}

For single photon emission computed tomography (SPECT), the use of the Jaszczak (Deluxe) phantom for overall system performance assessment is recommended by the European Association of Nuclear Medicine $(\mathrm{EANM})^{(6)}$, but also by the American Association of Physicists in Medicine (AAPM) and the American College of Radiology.

In this protocol, the phantom was filled with 400 $\mathrm{MBq}$ of ${ }^{99 \mathrm{~m}} \mathrm{Tc}$. Uniformity of the reconstructed slices, cold sphere contrast and spatial resolution based on the visualisation of cold rods were evaluated. The presence of macroscopic artefacts was also checked by visual inspection of the reconstructed images.

\section{Positron emission tomography}

For quantitative PET performance assessment, the EANM promoted EARL protocol (http://earl.eanm. org/cms/website.php?id=/en/projects/fdg_pet_ct_ accreditation.htm) was adopted as a reference. This protocol is based on a PET/CT acquisition of the NEMA-IEC body phantom filled with an ${ }^{18} \mathrm{~F}-\mathrm{FDG}$ activity concentration ratio of $10: 1$ between the hot spheres and the background, respectively. However, differently from the EARL protocol, the two largest 


\section{N. RYCKX ET AL.}

spheres were filled with plain water (no activity) to test cold contrast and, indirectly, the performance of scatter and attenuation corrections.

\section{CT use in NM and feedback}

Since there are currently no DRLs for the CT units used in SPECT/CT and PET/CT, the use of CT has to be evaluated in the scope of the different imaging purposes. Indeed, the dose requirements are not the same for a diagnostic scan than for the creation of a correction attenuation map. Furthermore, the technology employed in NM can differ from state of the art radiologic CT (e.g. cone-beam $\mathrm{CT}$ or a pseudo-CT with common gantry with the cameras). Therefore, CT imaging in NM examinations has to be correctly assessed based on the specific tasks to be accomplished.

For both imaging modalities (SPECT/CT and $\mathrm{PET} / \mathrm{CT}$ ), the local acquisition protocols were tested, results were discussed and a specific advice was given in order to optimise the image acquisition and/or reconstruction protocols to improve both qualitative and quantitative outcomes.

\section{Continuous education}

The collected data were summarised and shared with the users during continuous education (CE) sessions. The aim was to update the users' knowledge in radiation protection, optimise the clinical use of the devices, as well as to promote a team collaboration spirit.

\section{RESULTS}

\section{Computed tomography}

Forty-five CT units were tested in $2013(\sim 18 \%$ of all CT units in the country). Several elements were noticed.

\section{Machine output}

Four devices evidenced patient positioning laser alignment off by as much as $10 \mathrm{~mm}$, possibly resulting in an increase in patient dose of $6 \%$.

Nine of 45 CT units $(20 \%)$ presented a beam collimation that was widely different from the value stated by the device, e.g. $4 \mathrm{~mm}$ measured for $1 \mathrm{~mm}$ nominal. These units took this parameter into account into their beam efficiency calculation. For example, for a 1-mm single-slice acquisition protocol (e.g. examination of the posterior fossa or the petrous bone), one unit displayed an efficiency of $14 \%$, thus resulting in a CTDI $\mathrm{vol}_{\mathrm{vol}}$ of 299 $\mathrm{mGy}$, whereas a same-generation CT unit with a beam collimation of $1.25 \mathrm{~mm}$ with the exact same parameters displayed a $\mathrm{CTDI}_{\mathrm{vol}}$ of $41.5 \mathrm{mGy}$ for a beam efficiency of $55 \%$. Radiographers were not aware of the importance of their choice of collimator settings.

Twenty-one of $45 \mathrm{CT}$ units had $\mathrm{HU}$ in water that were not within the legal tolerance $(0 \pm 4 \mathrm{HU})$ for tube voltages different from 120 or $140 \mathrm{kV}$. This was mainly due to a lack of calibration because the tensions were not used in routine. Surprisingly, even then, an acquisition at a non-calibrated tension was still available in user mode, thus potentially leading to a completely erroneous examination if, for example, a newly engaged radiologist were to perform an examination at a lower tube voltage.

\section{Abdomen protocol qualification}

The subjective estimation of the LCD performance yielded some interesting results. For 2-mm slices, 4 of $16 \mathrm{CT}$ units showed a lower detection performance, whereas for the $2.5-\mathrm{mm}$ slices, 3 of $27 \mathrm{CT}$ units showed LCD performance under the mean value. The mean values are given in Table 1 . Without clearly setting one manufacturer apart from the others, it reminded the users that, for the same acquisition parameters, an increase in the slice thickness could lead to an increased LCD.

However, one of the main limitations of the authors' approach was that this was subjectively assessed, without taking into account the spatial frequency of the noise.

\section{Clinical use of the device}

The clinical use of the devices showed a large variability, especially for the abdominal examinations. Tables 2 and 3 and Figure 2 give an overview of this dispersion. For example, the ratio of the 75th and 25th percentile of the mean $\mathrm{CTDI}_{\mathrm{vol}}$ was 1.9 , whereas the effective dose showed a dispersion factor of 3.0. This shows that the number of scans per examination is not uniform.

In 2008, the FOPH estimated the mean effective dose for abdominal examinations at $\sim 9 \mathrm{mSv}$. The current analysis shows a value of $18.7 \pm 12.7 \mathrm{mSv}$, more than twice the last value (Figure 2 and Table 2). Reasons for this could be a miscalculation of the 2008 value, a quick increase in the number of scans per examination during this period or a biased selection of the examinations.

One critical aspect is that, among CT users - and MPEs - the concept of DRL is not clear, especially since the number of scans is not standardised. The current approach allowed for a clarification of the

Table 1. LCD performance for the unit benchmarking protocol.

\begin{tabular}{lcc}
\hline $\begin{array}{l}\text { Slice thickness } \\
{[\mathrm{mm}]}\end{array}$ & $\begin{array}{c}\text { LCD } @ 0.5 \% \\
{[\mathrm{~mm}]}\end{array}$ & $\begin{array}{c}\text { LCD } @ 0.3 \% \\
{[\mathrm{~mm}]}\end{array}$ \\
\hline 2 & $9.6 \pm 2.8$ & $14.5 \pm 1.7$ \\
2.5 & $8.0 \pm 0.6$ & $14.0 \pm 2.3$ \\
\hline
\end{tabular}

LCD performance is given in terms of the average smallest low-contrast insert detected, for a given native contrast $(0.5$ or $0.3 \%$ ). 
MEDICAL PHYSICS EXPERTS IN RADIOLOGY

Table 2. Mean values of the main dose indicators for clinical use of CT units, along with the measured ranges.

\begin{tabular}{lcccc}
\hline Protocol & Mean CTDI $_{\text {vol }}[\mathrm{mGy}]$ & Mean DLP $[\mathrm{mGy} \mathrm{cm}]$ & No. of phases & $E[\mathrm{mSv}]$ \\
\hline Head & $51.2 \pm 13.9$ & $905 \pm 275$ & $1.7 \pm 0.7$ & $3.2 \pm 1.3$ \\
& $22.1-84.2$ & $286-1812$ & $1-4$ & $1.0-6.6$ \\
Chest & $8.5 \pm 5.5$ & $310 \pm 165$ & $1.4 \pm 0.5$ & $6.2 \pm 3.5$ \\
& $2.6-31.8$ & $96-816$ & $1-3$ & $1.4-20.3$ \\
Abdomen & $12.2 \pm 7.0$ & $535 \pm 320$ & $2.4 \pm 1.3$ & $18.7 \pm 12.7$ \\
& $2.6-48.8$ & $96-2085$ & $1-9$ & $2.0-112.0$ \\
\hline
\end{tabular}

Mean CTDI ${ }_{\mathrm{vol}}$ and DLP are given per scan, whereas the effective dose and number of scans are given per examination.

Table 3. Dispersion of the clinical use in terms of the percentile of abdomen dose indicators.

\begin{tabular}{lccccr}
\hline Percentile & Mean CTDI vol $_{\text {valy }}[\mathrm{m}$ & Mean DLP $[\mathrm{mGycm}]$ & Cumulated DLP $[\mathrm{mGy} \mathrm{cm}]$ & No. of phases & $E[\mathrm{mSv}]$ \\
\hline 25 th & 8.0 & 320 & 550 & 1 & 8.4 \\
75 th & 14.8 & 665 & 1655 & 3 & 25.4 \\
75 th/25th & 1.9 & 2.1 & 3.0 & 3 & 3.0 \\
\hline
\end{tabular}

The dispersion of the practice is given by the ratio between the 75 th percentile and the 25 th percentile of the distribution, thus ignoring the extreme values.

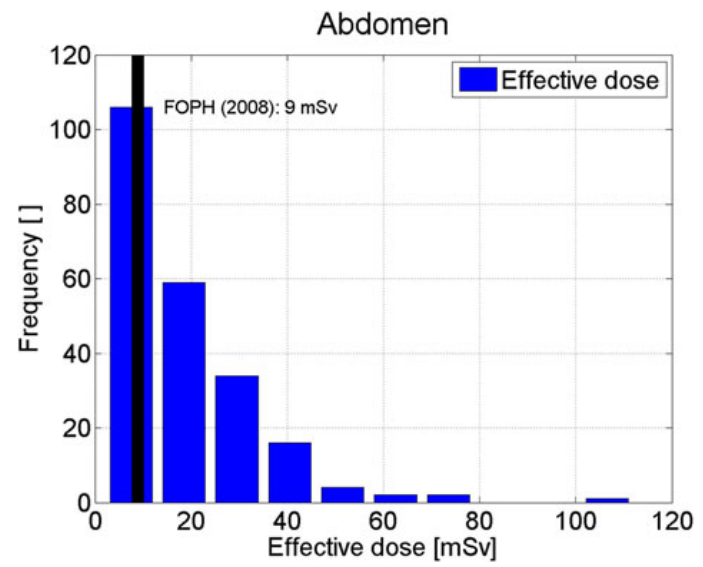

Figure 2. Dispersion of the clinical use of CT for abdominal examinations in terms of effective dose per examination. The 2008 value from the FOPH dose estimation is given as a comparison.

actual patient exposure, thus triggering discussions among radiologists in order to limit the dispersion of the practice.

A further limitation was the collection of clinical cases on the basis of anatomical regions. Thus, a bias was introduced by comparing examinations for obviously different clinical demands. An effort will be placed upon analysing clinical practice with respect to demand in the coming years.

\section{Fluoroscopy}

For approximately the same clinical settings, the dose rates showed large variations. For example, when putting $20 \mathrm{~cm}$ of PMMA in the primary beam, acquisitions at 15 frames per second (typical for interventional cardiology procedures) had differences up to a factor 3 (Figure 3). Measurements made with diagnostic settings (cineradiography or digital subtraction angiography) even yielded differences up to a factor 7 .

\section{Nuclear medicine}

The analysis of early results obtained from a few tested devices showed heterogeneous PET performance. The main factor for differences was the availability or not of time-of-flight and point spread function corrections that strongly impact both overall image noise and activity recovery in small-sized lesions.

Furthermore, although the radiographers practising SPECT/CT and/or PET/CT examinations were fairly aware of the principles of radiation protection when manipulating radioactive sources, they were not comfortable with the interpretation of the CT dose indicators, as there are still no existing DRLs specifically for transmission imaging in NM.

\section{Continuous education}

Approximately 150 radiographers have participated into $7 \mathrm{CE}$ sessions organised throughout the year. The lecture's themes were a description of the methodology 


\section{N. RYCKX ET AL}

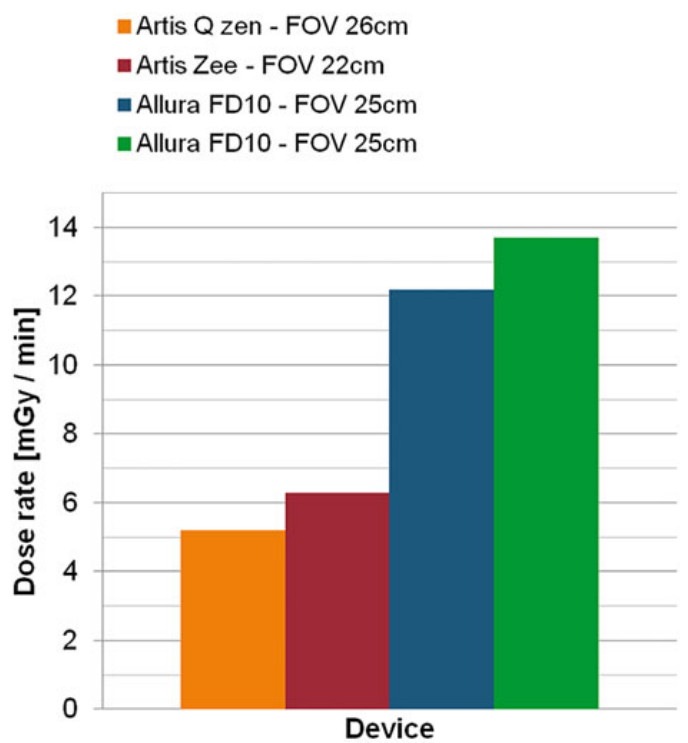

Figure 3. Discrepancy in the dose rates for different fluoroscopy units with the same acquisition settings (fluoroscopy, medium quality, 15 frames per second, $20 \mathrm{~cm}$ of PMMA in the primary beam, comparable FOV).

used in CT qualification within the new legal framework and a reminder of the dose indicators as well as the metrics of image quality with respect to dose. One important aspect was the gain of confidence between the radiographers and the MPE.

\section{PERSPECTIVES}

For CT, the future tasks are divided into two subsets. First, there is a strong need for an objective qualification of LCD, so the user is aware of the potential loss in LCD performance when using low-dose protocols along with iterative image reconstruction algorithms. Second, the sampling of examinations is to become task-oriented rather than relative to a given anatomical region. The ultimate goal would be to have one (or several) objective image quality indicator(s) for a given clinical demand. For example, the search of kidney stones (high contrast) does not need the same dose level than a suspected liver carcinoma. For this, the implementation of model observers on anthropomorphic systems is a promising tool towards this solution.

For fluoroscopy, a great deal of effort has been put into the establishment of new reference levels in interventional cardiology. For this purpose, automated dose collection software will become of prime importance. Nonetheless, a crucial point that has yet not been investigated thoroughly is the reliability of the dose indicators of the devices, such as cumulative dose or cumulative dose-area product. Indeed, the former is defined at two different locations in the beam (FDA or IEC reference points); the latter, especially since diagnostic beams are heavily filtered using copper, is, based on preliminary measurements, potentially off by a factor of 2. A new investigation protocol to address this problem is currently being set up by the AAPM task group 190, and their final report is due in December 2014. As a consequence, the traceability of the testing instruments will be even more important, and the tolerance on dose indicators will have to be lowered. Furthermore, the nomenclature of the specific diagnostic and/or therapeutic interventions is not unified, thus making a proper large-scale dose collection tedious. Interventional cardiology, for which soon-to-be published DRL have been established (as cited previously), could serve as a role model for this task. An effort will also be taken on the establishment of a database of objective image quality indicators for parameters deemed acceptable by physicians after optimisation.

Finally, for NM, two aspects seem to emerge from the first results. First, a great deal of effort has to be put into the calibration of the individual activity meters of the different institutions, by using a solid ${ }^{68} \mathrm{Ga} /{ }^{68} \mathrm{Ge}$ phantom for PET and the Jaszczak phantom for SPECT. Second, the use of CT for attenuation correction will, as for conventional CT, benefit of the model observer routines to tackle objective image quality.

The CE sessions have allowed the MPE to gain knowledge of the devices across the country and confidence with respect to their clinical applications. The next step will be to establish partnerships with radiologists.

\section{REFERENCES}

1. Swiss Federal Council. Radiological Protection Ordinance (RPO) of 22 June 1994, classified compilation 814.501. Swiss Federal (2008).

2. Deak, P. D. et al. Multisection CT protocols: Sexand age-specific conversion factors used to determine effective dose from dose-length product. Radiology 257, 1 (2010).

3. Paolicchi, F. et al. Real practice radiation dose and dosimetric impact of radiological staff training in body CT examinations. Insights Imaging, 4(2), 239-2441 (2013).

4. AAPM Report 204, Size-Specific Dose Estimates (SSDE) in pediatric and adult CT examinations. ISBN: 978-1-936366-08-8, AAPM (2011).

5. Mesbahi, A. et al. Comparison of radiation dose to patient and staff for two interventional cardiology units: a phantom study. Radiat. Prot. Dosim. 131(3), 399-403 (2008).

6. Routine quality control recommended for nuclear medicine instrumentation. Eur. J. Nucl. Med. Mol. Imaging 37, 662-671 (2010).

7. Habibzaden, M. A. et al. Impact of miscentering on patient dose and image noise in $x$-ray CT imaging: phantom and clinical studies. Physica. Medica. 28, 191-199 (2012). 